\title{
AC charging/discharging of de-alloyed Si-AI-V alloy ribbons
}

\author{
M. Fukuhara ${ }^{\mathrm{a}, \mathrm{b},{ }^{*}}$ and H. Yoshida ${ }^{\mathrm{a}, \mathrm{b}}$ \\ a Institute for Materials Research, Tohoku University, Sendai 980-8577, Japan \\ b Research Institute for Electromagnetic Materials, Sendai 982-0807, Japan \\ *Corresponding author. Tel.: +81-22-245-8513; fax: +81-22-245-8031 \\ E-mail address: fukuhara@cd.wakwak.com (M. Fukuhara)
}

\begin{abstract}
In anticipation of prompt ac charging/discharging characteristics, the capacitance of de-alloyed $\mathrm{Si}_{1-\mathrm{x}}\left(\mathrm{Al}_{0.5}, \mathrm{~V}_{0.5}\right)_{\mathrm{x}}(\mathrm{x}=0.15,0.2,0.3$, and 0.4$)$ alloy ribbons with resistivities of $0.01-4300 \Omega \mathrm{cm}$ was measured as a function of frequency between $1 \mathrm{mHz}$ and $1 \mathrm{MHz}$, using exponential transient analysis for electric charging/discharging. The capacitance of de-alloyed Si-7.5 at $\% . A 1-7.5$ at $\%$. V obtained by prompt (100 ms-25 ns) charging/discharging decreased logarithmically from $105 \mu \mathrm{F}$ to $18 \mathrm{pF}$ as frequency increased from $1 \mathrm{mHz}$ to $10 \mathrm{kHz}$, and then saturated between $10 \mathrm{kHz}$ and $1 \mathrm{MHz}$. From the observed electrode distance dependence on capacitance, we deduced that the alloy consisted of a distributed constant equivalent circuit (series with $1.2 \%$ parallel), analogous to electric double layer capacitors.
\end{abstract}

Keywords: metals and alloys, rapid-solidification, quenching, dielectric response, capacitance spectroscopy 


\section{Introduction}

Generally, electric charge cannot be stored in metals (or alloys) without a dielectric open space. The ability to store charge in a centimeter-sized glassy alloy without dielectric open space, would lead to significant advancements in the field of not only electronic devices but also electric power applications. Recently, we found that the $\left(\mathrm{Ni}_{0.36} \mathrm{Nb}_{0.24} \mathrm{Zr}_{0.40}\right)_{90} \mathrm{H}_{10}$ glassy alloy, showing Coulomb dot oscillation at room temperature [1] and a semi-true circular Nyquist diagram with total capacitance of 17.8 $\mu \mathrm{F}[2]$, can be regarded as a dc/ac converting device having a large number of 0.23-nm-capacitors with femtofarad capacitance among its distorted icosahedral $\mathrm{Zr}_{5} \mathrm{Ni}_{5} \mathrm{Nb}_{3}$ clusters (dots of ca. $0.55 \mathrm{~nm}$ in size [3]). In this material, hydrogen atoms flow into spaces outside the clusters, enlarging the spaces and resulting in the construction of zigzag tunnels of $0.23 \mathrm{~nm}$ average width because of the high pressure effect of hydrogen during electrolysis [4]. However, severe constraints on prompt discharging were observed experimentally for this material, because the electric charge behaves as a polarized glutinous liquid that is absorbed between pairs of metallic clusters [5]. This is known as electroadsorption [6]. In practice, we found that the low resistance of the Ni-Nb-Zr-H glassy alloy in the input circuit prevented its prompt discharging to an output circuit with larger resistance. Rapid, powerful, and energy-rich 
storage effects in such materials are necessary for future electronic devices and electric power applications such as hybrid electric vehicles and backup power supplies.

In anticipation of prompt charge/discharging, we reported electric storage characteristics for de-alloyed Si-Al alloy ribbons with a capacitance of $102 \mu \mathrm{F}$ at $1 \mathrm{mHz}$ [7]. We found that the de-alloyed specimen had an electronic transport structure similar to that of a metal-semiconductor junction (i.e. Schottky junction) [8], which is attributable to the residue of $\mathrm{Al}$ in the $\mathrm{Si}$ skeleton. For the fractal structure of the de-alloyed Si-Al alloy capacitor, we assumed that the structure resembles that of an electric double-layer capacitor (EDLC), which is composed of a distributed constant equivalent circuit of active carbon $(R)$ and electrolyte $(C)[9,10]$. The Si skeleton and Al backbone is to an electron what active carbon is to the electrolyte solution in EDLC. However, the transport rate of the electrons contributes to prompt charging/discharging, gaining an advantage over conventional batteries such as EDLC.

In this study, Si-Al-V alloys were chosen as starting alloys for the formation of nanometer-sized porous structures with high resistivities of $10 \mu \Omega \mathrm{cm}$ to $4.3 \mathrm{k} \Omega \mathrm{cm}$ using a de-alloying method, with an aim to obtain further rapid charge/discharge characteristics rather than those of de-alloyed Si-Al alloys. 


\section{Experimental}

Si-Al-V alloy ingots (compositions given in nominal atomic percentage) were prepared by arc-melting mixtures of Si (99.999 \% purity), Al (99.95\% purity), and V (99.9 \% purity) in an argon atmosphere purified by Ti- gettering. Ribbon samples of 30 $60 \mu \mathrm{m}$ in thickness and 1-2 $\mathrm{mm}$ in width were prepared from these ingots by rapid solidification of the melt on a single copper roller at a tangential velocity of $31.4 \mathrm{~m} / \mathrm{s}$. De-alloying of the samples was carried out for $259.2 \mathrm{ks}$ in $1 \mathrm{~N} \mathrm{HCl}$ solution to remove the $\mathrm{Al}$ content and then for $86.4 \mathrm{ks}$ in condensed $\mathrm{HNO}_{3}$ to eliminate the $\mathrm{V}$ content, at room temperature.

The sample structure was examined by X-ray diffraction (XRD, CN4012A1, Rigaku, Tokyo, Japan) in reflection mode with monochromatic $\mathrm{Cu} \mathrm{K \alpha}$ radiation. Surfaces of the de-alloyed specimens were examined by scanning electron microscopy (SEM, Hitachi S3800, Tokyo, Japan).

Electrical resistances of the Si-Al-V alloys before and after de-alloying were measured at room temperature using a four-terminal measuring method with electrode distances of 2-20 mm. Capacitances were calculated as a function of frequency between $1 \mathrm{mHz}$ and $1 \mathrm{MHz}$ from electric charge/discharge pulse curves of $10 \mathrm{~V}$ applied at $25 \mathrm{~ns}-$ $100 \mathrm{~ms}$ intervals, using a mixed-signal oscilloscope (MSO 5104, Tektronix, OR, USA) 
and $30 \mathrm{MHz}$ multifunction generator (WF1973, NF Co.Yokohama, Japan) on the basis of simple exponential transient analysis.

\section{Results and discussions}

To select alloys with charging/discharging abilities suitable for electric storage capacitors, we measured the electrical resistivity at room temperature of the $\mathrm{Si}-(\mathrm{Al}+\mathrm{V})$ alloys before and after acid leaching. The results are shown in Fig. 1a, along with reference data for $\mathrm{Si}-\mathrm{V}$ [11], -Ce [12], -Nb [13], and -AlFeNiCr [14] alloys. The observed resistivity change of the $\mathrm{Si}-(\mathrm{Al}+\mathrm{V})$ alloys before acid leaching is fairly consistent with that reported for $\mathrm{Si}-\mathrm{V},-\mathrm{Ce},-\mathrm{Nb}$, and $-\mathrm{AlFeNiCr}$ alloys. The resistivity of the de-alloyed samples decreased from $10^{9}$ to $10^{4} \mu \Omega \mathrm{cm}$, values $10^{-2}-10^{-7}$ lower than that of $\mathrm{Si}\left(2 \times 10^{11} \mu \Omega \mathrm{cm}\right)$ with increasing $(\mathrm{Al}+\mathrm{V})$ content. In anticipation of the higher resistivity required for prompt discharging, we selected the Si-7.5 at.\% Al-7.5 at.\% V sample as the representative specimen in this study. Fig. 1(b) and (c) show XRD spectra of the specimens before and after de-alloying, respectively. Before de-alloying, the sample consists of a mixture of crystalline $\mathrm{Si}, \mathrm{Al}, \mathrm{VSi}_{2}, \mathrm{Si}_{2} \mathrm{~V}$, and $\mathrm{Al}_{2} \mathrm{O}_{3}$, while after de-alloying the sample exhibited polycrystalline $\mathrm{Si}$, also with low $\mathrm{Al}_{2} \mathrm{O}_{3}$ content.

We then measured voltage transient phenomena as a function of time for charging and 
discharging in input and output circuits at $1 \mathrm{kHz}$. The results are presented at Fig. 2(a) and (b). Transient analysis of Fig. 2(a) and (b) gave capacitance values of 77 and $111 \mathrm{pF}$ for charging and discharging, respectively. Capacitance as a function of frequency for this sample is presented logarithmically in Fig. 2(c). All capacitances decreased parabolically to $18 \mathrm{pF}$ with increasing frequency up to $10 \mathrm{kHz}$, before becoming saturated in the frequency region $10 \mathrm{kHz}$ to $1 \mathrm{MHz}$. The alloy showed a maximum capacitance of $105 \mu \mathrm{F}$ for discharging at $1 \mathrm{mHz}$, being almost the same as de-alloyed Si-Al alloys. This behavior implies ac current charging/discharging, with the observed decrease in capacitance derived from dielectric interfacial dispersion at higher frequencies.

As can be seen from the resistivity in Fig. 1(a) and capacitance in Fig. 2(c), the capacitance is associated with resistivity of de-alloyed specimens after leaching. The effect of the resistivity on the capacitance of the de-alloyed Si-7.5 at. \%Al-7.5 at. \%V, -10 at. $\% \mathrm{Al}-10$ at. $\% \mathrm{~V}$, and -15 at. $\% \mathrm{Al}-15$ at. $\% \mathrm{~V}$ specimens is presented in Fig. 3, along with those of the $\mathrm{Si}-20$ at. $\% \mathrm{Al},-30$ at. $\% \mathrm{Al}$, and -40 at. $\% \mathrm{Al}$ specimens [7]. Capacitance increases with increasing resistivity. This suggests higher capacitance needs for higher resistivity. Furthermore, the addition of $\mathrm{V}$ enhances the capacitance for the de-alloyed Si-Al specimens. 
Since it is considered that de-alloyed $\mathrm{Si}-(\mathrm{Al}+\mathrm{V})$ is organized as a complex circuit of an $m$-rank parallel and $n$-row series combination of femtofarad Si capacitors, as is the case for $\mathrm{Si}-\mathrm{Al}$ [7] alloys and $\mathrm{Ni}-\mathrm{Nb}-\mathrm{Zr}-\mathrm{H}$ glassy alloys [2], we measured the electrode distance dependence on discharging capacitance for the de-alloyed specimen. As can be seen from Fig. 4(a), the capacitance $C$ for all frequencies increased linearly with electrode distance $d$. At $0.1 \mathrm{~Hz}, C=3.06 d+969.7$ (Fig. 4(b)), suggesting an existence of parallel circuits in the alloy. However, if a perfect parallel combination existd, the capacitance would be expressed by $C=254.8 d$ and the alloy would be composed of distributed constant equivalent circuits of series $C$ with $1.2 \%$ parallel $C$ (Fig. 4(c)). Figure 4(d) presents the surface microstructure of the de-alloyed specimen prepared in $\mathrm{HCl}$ and $\mathrm{HNO}_{3}$ solutions, showing $\mathrm{Si}$ grains with narrow canyons which store electric charge. Although an investigation of the three-dimensional cluster configuration is indispensable to the construction of giant capacitance, it was very difficult to determine the actual canyon structure because of its brittleness. Further precise observation is called for. Since large electric storage could be obtained by the parallel combination network of huge numbers of atomic-size spaces, further improvemenst are also required such as supercooling through higher roller speeds [5] and hydrogen penetration [1-4] for construction of surface structures with a higher density of extremely narrow canyons. 


\section{Conclusion}

To improve the capacitance of de-alloyed Si-Al alloys, the frequency dependent capacitance of de-alloyed $\left.\mathrm{Si}_{1-\mathrm{x}(} \mathrm{Al}_{0.5}, \mathrm{~V}_{0.5}\right)_{\mathrm{x}}(\mathrm{x}=0.15,0.2,0.3$, and 0.4$)$ alloy ribbons was measured between $1 \mathrm{mHz}$ and $1 \mathrm{MHz}$ at room temperature, using exponential transient analysis for electric charging/discharging. The de-alloyed specimens showed resistivities from $0.01-4300 \Omega \mathrm{cm}$, values $10^{-2}-10^{-7}$ lower than that of Si $\left(2 \times 10^{11}\right.$ $\mu \Omega \mathrm{cm})$ with increasing $(\mathrm{Al}+\mathrm{V})$ content. The $\mathrm{Si}-7.5$ at. $\% \mathrm{Al}-7.5$ at. $\% \mathrm{~V}$ alloy ribbon showed maximum capacitance of $105 \mu \mathrm{F}$ for discharging at $1 \mathrm{mHz}$. All capacitances decreased parabolically with increasing frequency up to $10 \mathrm{kHz}$, before becoming saturated in the frequency region $10 \mathrm{kHz}$ to $1 \mathrm{MHz}$. In comparison with de-alloyed Si-Al and Si-Al-V specimens, the addition of $\mathrm{V}$ enhances the capacitance. A higher capacitance requires a higher resistivity of the de-alloyed specimens. From the electrode distance dependency for the capacitance, the alloy would be composed of distributed constant equivalent circuits of series $C$ with $1.2 \%$ parallel $C$. Thus this paper provides useful information for novel science and technologies associated with battery materials.

\section{Acknowledgments}

This work was supported by a Grant-in-Aid for Science Research in a Priority Area, 
'Advanced Materials Development and Integration of Novel Structured Metallic Glasses and Inorganic Materials', from the Ministry of Education, Culture, Sports Science and Technology, Japan.

\section{References}

[1] M. Fukuhara, A. Inoue, Room-temperature Coulomb oscillation of a oroton dot in Ni-Nb-Zr-H glassy alloys with nanofarad capacitance, J. Appl. Phys. 105 (2009) 063715.

[2] M. Fukuhara, M. Seto, A. Inoue, ac impedance analysis of a Ni-Nb-Zr-H glassy alloy with femtofarad capacitance tunnels, Appl. Phys. Lett. 96 (2010) 043103.

[3] M. Fukuhara, N. Fujima, H. Oji, A. Inoue, S. Emura, Structures of the icosahedral clusters in $\mathrm{Ni}-\mathrm{Nb}-\mathrm{Zr}-\mathrm{H}$ glassy alloys determined by first-principles molecular dynamics calculation and XAFS measurements, J. Alloy. Comp. 497 (2010) 182-187.

[4] M. Fukuhara, H. Yoshida, K. Koyama, A. Inoue, Y. Miura, Electronic transport behaviors of Ni-Nb-Zr-H glassy alloys, J. Appl Phys. 107 (2010) 033703.

[5] M. Fukuhara, H. Yoshida, N. Fujima, H. Kawarada, Capacitance Distribution of Ni-Nb-Zr-H Glassy Alloys, J. Noanosci. Nanotech., 12 (2012) 3848-3852.

[6] M. Dupeyrat, E. Nakache, Electrocapillarity and Electroadsorption, J. Colloid Interface Sci. 73 (1980) 332-344.

[7] M. Fukuhara, T, Araki, K. Nagayama, H. Sakuraba, Electric storage in de-alloyed Si-Al ribbons, Europhys. Lett., 99 (2012) 47001.

[8] H. Tsuji, N. Arai, T.Matsumoto, K. Ueno, Y. gotoh, K.Adachi, H. Kotaki, J.Ishikawa, 
Silver nanoparticle formation in thin oxide layer on silicon by silver-negative-ion implantation for Coulomb blockade, Appl. Surf. Sci. 238 (2004) 132-137.

[9] M. Itagaki, S. Suzuki, I. Shitanda, K. Watanabe, H. Nakazawa, Impedance analysis on electric double layer capacitor with transmission line model, J. Power Source, 164 (2007) 415-424.

[10] M. Okamura, Electric Double Layer Capacitor and Its Storage System, Nikkan Kogyo, Tokyo, 2011, P. 36.

[11] U. Mizutani, T. Ishizuka, and T. Fukunaga, J. Phys. Cond. Mat. 9 (1997) 5333.

[12] T. Biwa, M. Yui, T. Takeuchi, and U. Mizutani, Metal-Insulator Transition in the Amorphous $\mathrm{CexSi}_{100}-\mathrm{x}(4 \leq \mathrm{x} \leq 83)$ Heavy Fermion System, Mater.Trans. 42 (2001) 939-950.

[13] G. Hertel, D.J. Bishop, E.G. Spencer, J.M. Rowell, R.C. Dynes, Tunneling and Transport Measurements at the Metal-Insulator Transitiopn of Amorphous Nb:Si, Phys. Rev. Lett. 50 (1983) 743-746 .

[14] D.V.Louzguine-Luzgin, A. Inoue,metal ${ }^{\wedge}$ Semiconductor Amorphous and Nanoscale Ge-Phase Composites Produced by Rapid Solidification and by Devitrification of an Amorphous Matrix, J. Electro. Mater., 35 (2006) 19861992.

\section{Caption}

Fig. 1 (a) Eectrical resistivities for Si-15,-20,-30 and -40 at. \% (Al+V) alloys before and after $\mathrm{HCl}$ and $\mathrm{HNO}_{3}$ leaching, and $\mathrm{Al}-\mathrm{V}$ [11], -Ce [12], -Nb [13], and -AlFeNiCr [14] alloys, and X-ray diffraction spectra before (b) and after (c) $\mathrm{HCl}$ and $\mathrm{HNO}_{3}$ leaching for Si-7.5 at. \% Al-7.5 at. \% V. 
Fig. $2(a, b)$ Voltage transient curves for charging and discharging in the input and output circuits at $0.1 \mathrm{~Hz}$. (c) Frequency dependence of capacitance for Si-15, -20, $-30,-40$ at. $\%(\mathrm{Al}+\mathrm{V})$.

Fig. 3 Relation between capacitance and resistivity for de-alloyed Si-Al-V and Si-Al specimens.

Fig. 4 (a, b) Electrode distance dependence of capacitance, (c) Complex circuit of m-rank parallel and n-raw series combination in glassy alloys . (d) SEM image of de-alloyed Si-7.5 at. \% Al -7.5 at. $\%$ V alloy.
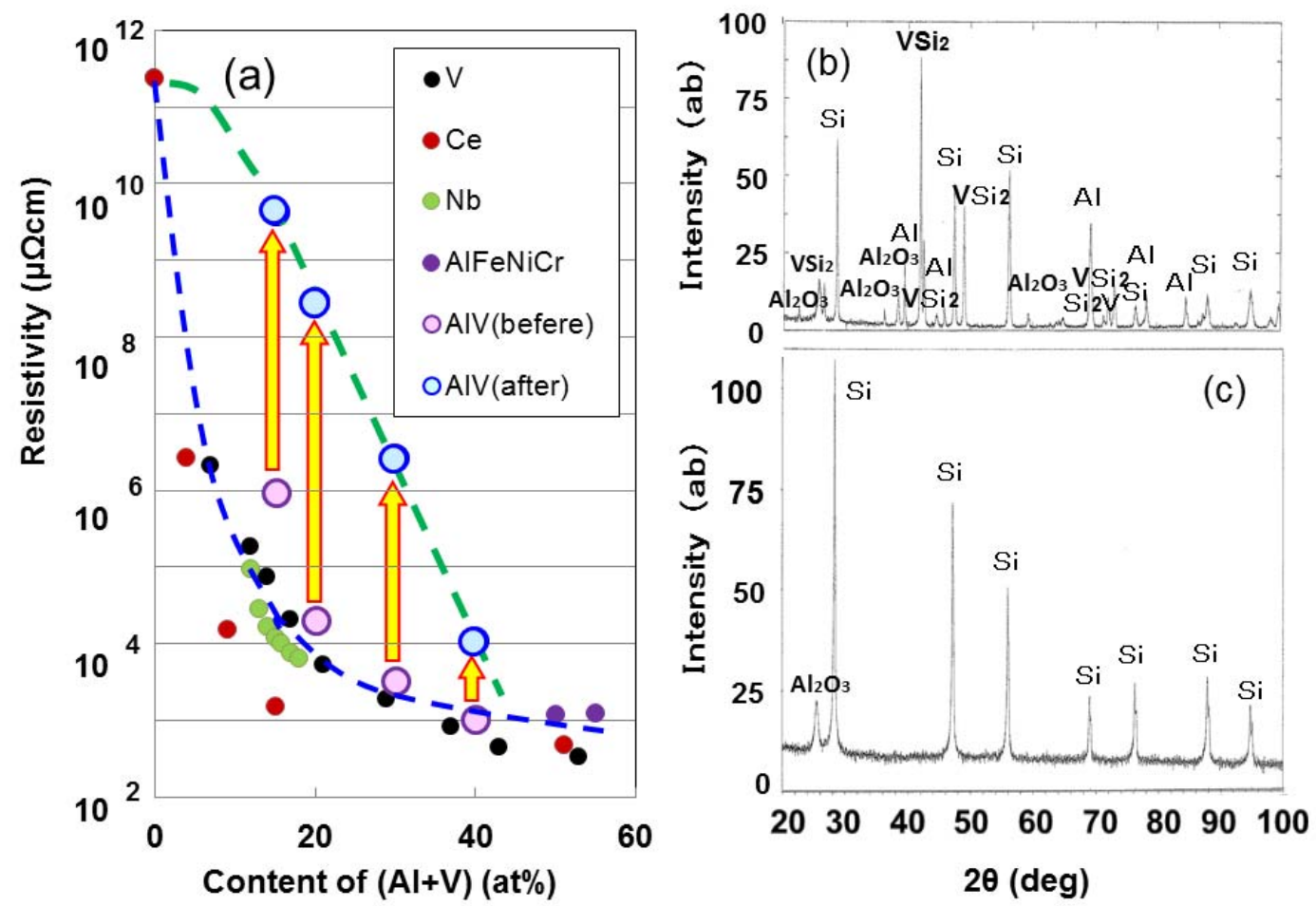

Figure 1 

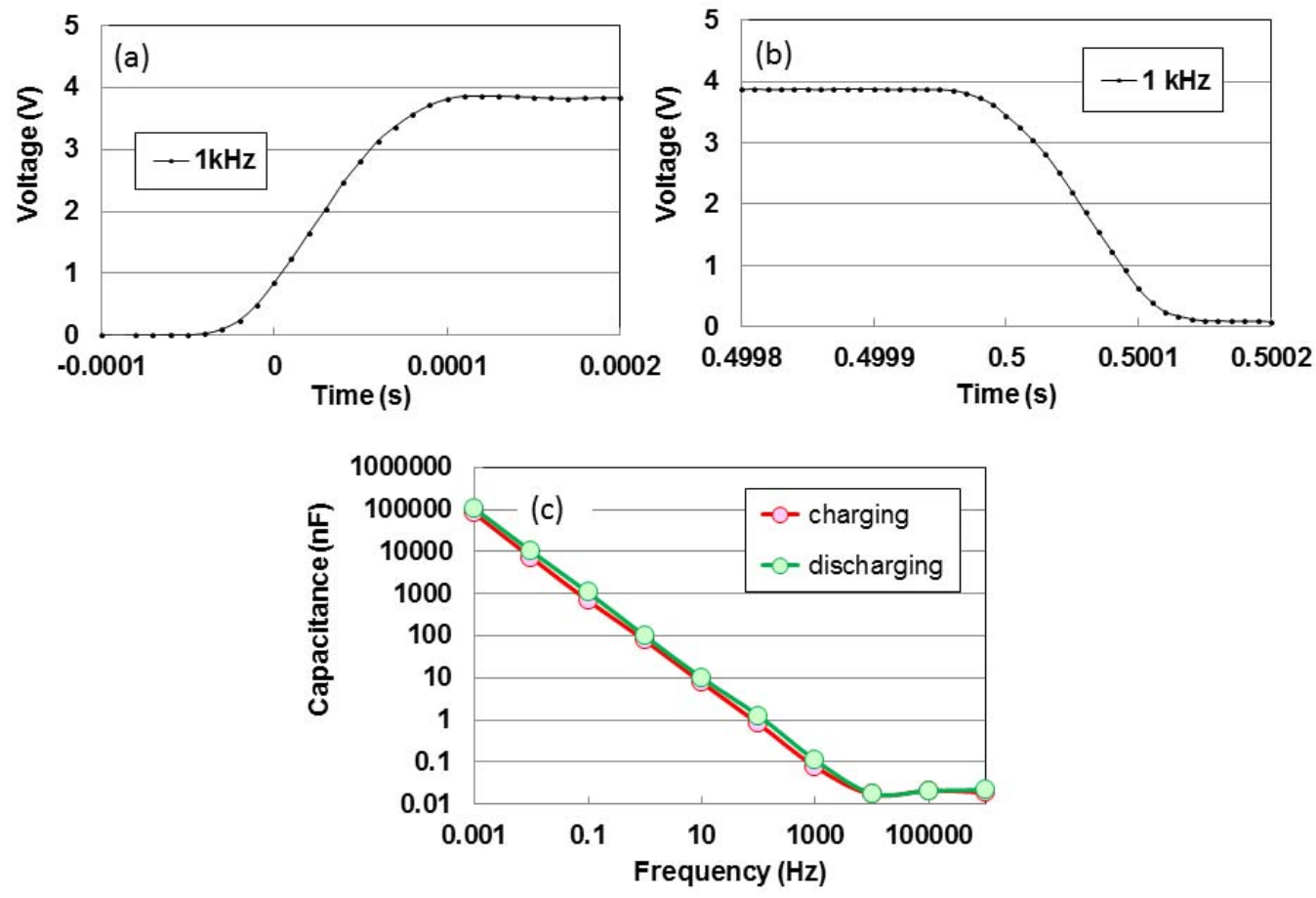

Figure 2 


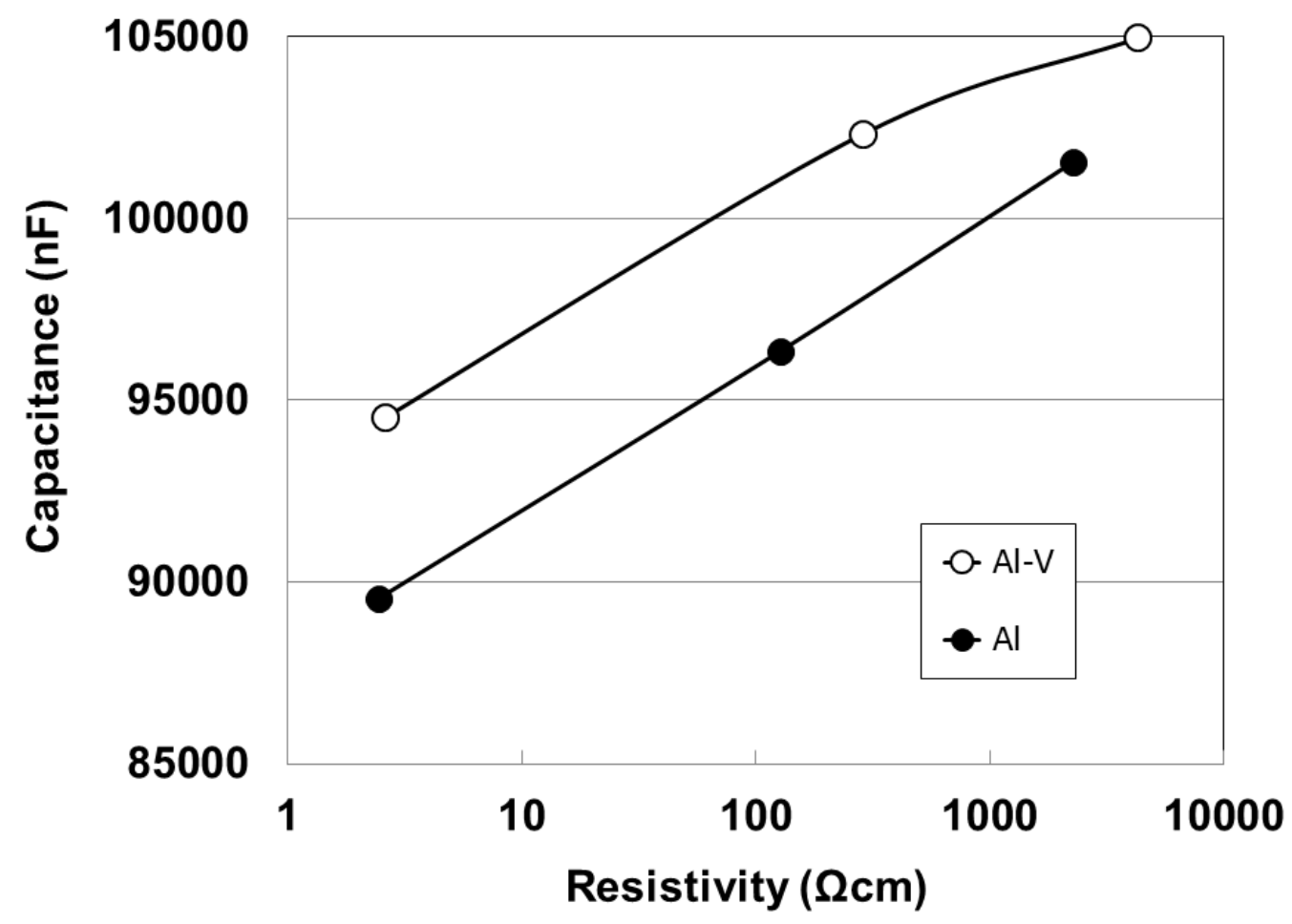

Figure 3 

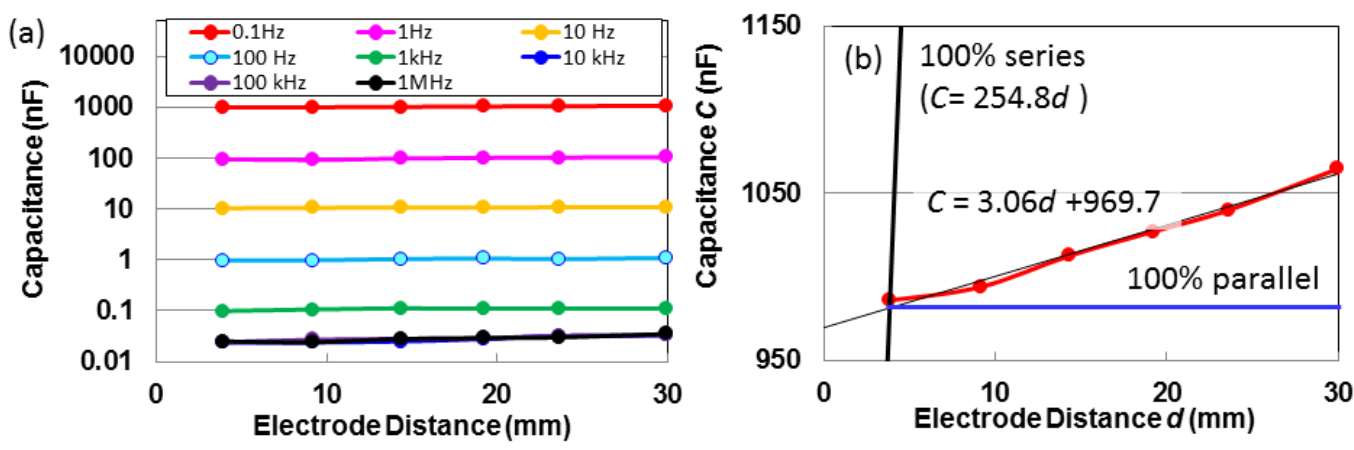

(c)
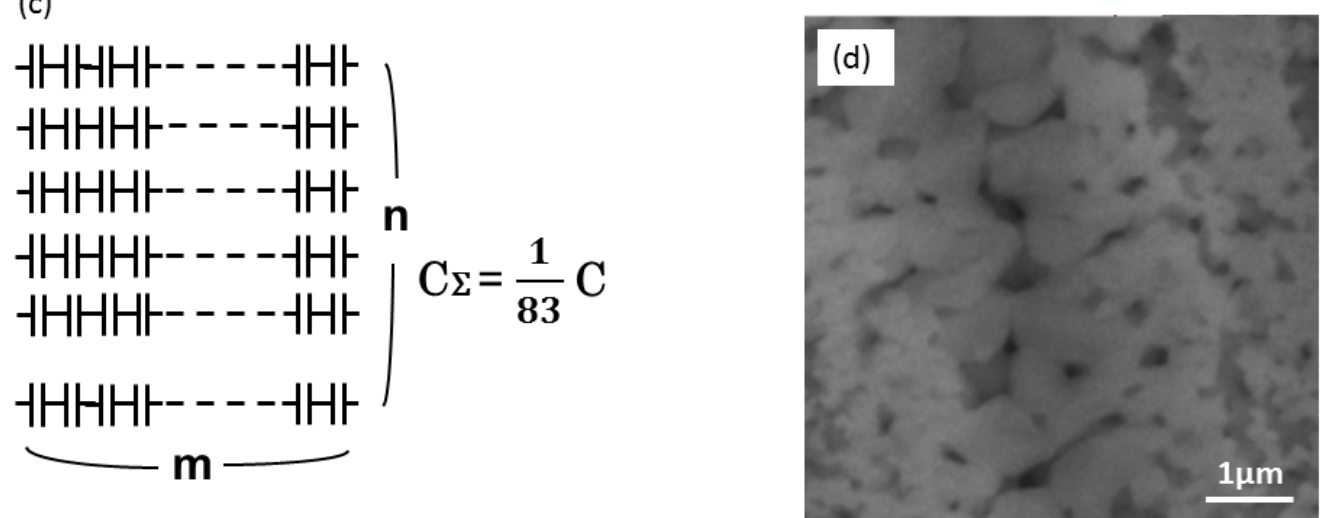

Figure 4 